\title{
Intermediate Product Profile as a Result of Ozone Oxidation of Industrial Wastewater
}

\author{
Ali Rıza DINÇER ${ }^{1}$ İbrahim Feda ARAL ${ }^{2 *}$ \\ 1.Department of Environmental Engineering, Namik Kemal University, Corlu, Tekirdag, Turkey \\ 2.Department of Civil Engineering, Namık Kemal University, Çorlu, Tekirdağ, Turkey
}

\begin{abstract}
The following were found when testing the wastewater in the study: 0.38\% 7-Hydroxy-7-phenly-3,9-diisopropyl2,10-dioxadispiri, 1.71\% 2-Propanone (CAS) Acetone, 20.87\% 2-propanol, 2-methyl- (CAS) tert-Butyl alcohol, 14.92\% 2-Butanone (CAS) Methyl ethyl ketone, 62.11\% Hydroperoxide, 1,1-dimethyl (CAS) Cadox TBH, COD $(10,000-23,725 \mathrm{mg}$ COD / 1), total kjeldahl nitrogen $(<6.2 \mathrm{mg} / \mathrm{l})$, free chlorine $(<0.05 \mathrm{mg} / \mathrm{l})$ and chloride $(>$ $9,042 \mathrm{mg} / \mathrm{l})$. The water was then oxidated using ozone $(4.1 \mathrm{~g} / \mathrm{h})$ and intermediate product formation and COD removal efficiency were studied. The current wastewater characteristics vary in line with product supply and demand.In this study, a cylinder reactor with an internal diameter of $5.8 \mathrm{~cm}$ and a height of 1.7 meters was used for ozonation ( $4.1 \mathrm{~g} / \mathrm{saat})$. After seven hours of ozonation, the wastewater $\mathrm{pH}$ value fell below four, showing that complex compounds were broken down to organic acids. At the end of the first, third, fourth, fifth and sixth hours of the ozonation process, respectively, nitrogen oxide (H), N, 2-Dimethydodecylamine (K), Propanoic acid, 2hydroxy-2-methyl (hydroxybutyric) (L), 2-Butanone, 3-hydroxy-3-methyl (M), 2,3 Butanedione (diacetyl) (N), Ammonium bicarbonate $(\mathrm{O}))$, Carbamicacid, monoammonium salt (Ammonium Carbamat) (P), Acetic acid, ethoxy-, 1-methylethyl ester (R) intermediates were formed. After seven hours of ozonation, the influent COD decreased from $17,000 \mathrm{mg} / 1$ to $2,860 \mathrm{mg} / 1$. As a result of the ozone process, the organic substances were mostly transformed into 2-propanone (Acetone) (B) and 2-propanol, 2-methyl (tert-butyl alcohol) (C).
\end{abstract}

Keywords: Ozonation, $\mathrm{COD}$, Industrial wastewater, Intermediate product

DOI: $10.7176 / \mathrm{CER} / 11-10-03$

Publication date: November $30^{\text {th }} 2019$

\section{Introduction}

In the treatment of industrial wastewater (containing high COD and low BOI), chemical oxidation methods and subsequent biological treatment systems are required. In the present study, industrial wastewater containing organic peroxide, high COD (higher than 10,000 mg/l), aldehyde, and ketone, which were difficult to break down, was analyzed.

Chemical oxidation is a widely used process for the treatment of water, including refractory compounds. Ozone is known to be a strong oxidant with the highest thermodynamic oxidation potential of common oxidants(Aparicio et al.,2007). As a chemical oxidation process, ozonation is a suitable system for decomposing pollutants and increasing the efficiency of biological treatments (Gomes et al.,2017). Ozone reacts quickly with electrophilic parts such as the chromophoric group of the azo bonds $(\mathrm{N}=\mathrm{N})$ ( De Souza et al., 2010). As ozone is a selective oxidation agent, the reactivity of different micro-pollutants with ozone is different (Hollender et al.,2009). Ozone is a strong oxidizing agent that breaks down compounds containing aromatic rings and double bonds. Thus, it can cause oxidative degradation of many organic compounds such as polyphenols. Depending on the $\mathrm{pH}$ value, the ozonation process follows two different paths. It is known that ozone oxidizes organic compounds by direct oxidation or by the production of hydroxyl radicals. Under acidic conditions, ozone reacts directly with organic compounds as an electrophile. Ozone attacks conjugated double bonds (Turhan et al.,2012). As a result, aldehydes, carboxylic acids and other by-products are formed (Asghar et al.,2015). Ozonation used in water and wastewater treatment results in the formation of various organic (carbonyl compounds) and inorganic by-products (Papageorgiou et al.,2017). Hydroxyl radicals are non-specific oxidizing agents that can degrade a number of contaminants because they react with almost all organic species. Reaction of molecular ozone with alkenes leads to the formation of an intermediate product, molozonide. The final products of this reaction are generally aldehydes, carboxylic acids, ketones and / or carbon dioxide (Gunukula et al.,2001). Reaction of ozone with hydrogen peroxide causes hydroxyl radical formation. This method has been successfully used in many applications. Since $\mathrm{HO}_{2}{ }^{-}$is highly reactive with ozone, the decomposition rate of ozone in the presence of hydrogen peroxide increases with $\mathrm{pH}$ (Gunukula et al.,2001).

Ozone is applied to wastewater treatment as a strong oxidizer. It reacts selectively with organic compounds at acidic $\mathrm{pH}$. Furthermore, ozone reacts slowly with certain organic substances such as inactivated aromatics or saturated carboxylic acids, and in most cases these organic compounds are not completely mineralized (Mehrjouei et al., 2015). Ozonation does not completely mineralize organic substances. Instead, it oxidizes them into more biodegradable forms (Lee et al.,2012). Ozonation is also a useful process in reducing toxicities in a wastewater stream. Pollutants such as phenols, cyanides, alcohols, pesticides, aldehydes and sulfides are successfully removed 
by ozonation (Gunukula et al.,2001). Ozone is a very strong oxidant that can react with a large number of chemicals $($ Eo $=+2.07 \mathrm{~V})$. As a result of the ozonation of some organic compounds, the biodegradability or toxicity of the oxidation products decreases(Gunukula et al.,2001).

After ozonation, an additional biological post-treatment is required to eliminate the negative ecotoxicological effects that occur during ozonation from biodegradable ozonation transformation products (OTPs) and oxidation by-products (OBPs) (Bourgin et al., 2018). The ozonation system alone cannot provide full mineralization. Therefore, a combination of ozonation and biodegradation has been proposed in a multistage system to improve cost and purification efficiency.

The main purpose of this research is to provide COD removal by oxidation with ozone of the wastewater of a factory producing organic peroxide and to reveal the formation and profile of the intermediate product. It has been determined that with ozone the organic matter has been transformed into the final oxidation products.

\section{Materials and Method}

In this study, COD exchange and intermediate product profile were examined as a result of ozonation of wastewater containing organic peroxide. The ozonation process was conducted with an ozone generator with a capacity of $4.1 \mathrm{~g} \mathrm{O}_{3} /$ hour. A stainless steel reactor with an internal diameter of $5.8 \mathrm{~cm}$ and a height of $1.7 \mathrm{~m}$ was used as the ozone reactor (Figure 1). Unused ozone in the system was maintained in wash bottles containing potassium iodide. The raw wastewater composition was determined using the gas chromatography and mass spectrometry (Shimadzu GCMS-QP2020) (Figure.1). During the seven-hour ozonation process, the product profile, $\mathrm{pH}$ and COD changes were observed. The ozone-treated wastewater was passed through the gas chromatography and mass spectrometry to determine the wastewater interim products and conversion rates. Figure 1 shows the experimental setup. The COD change interval ranges from 10,000 to 23,725 mg/l. The nitrogen concentration was generally low $(<6.2 \mathrm{mg} / \mathrm{l})$.

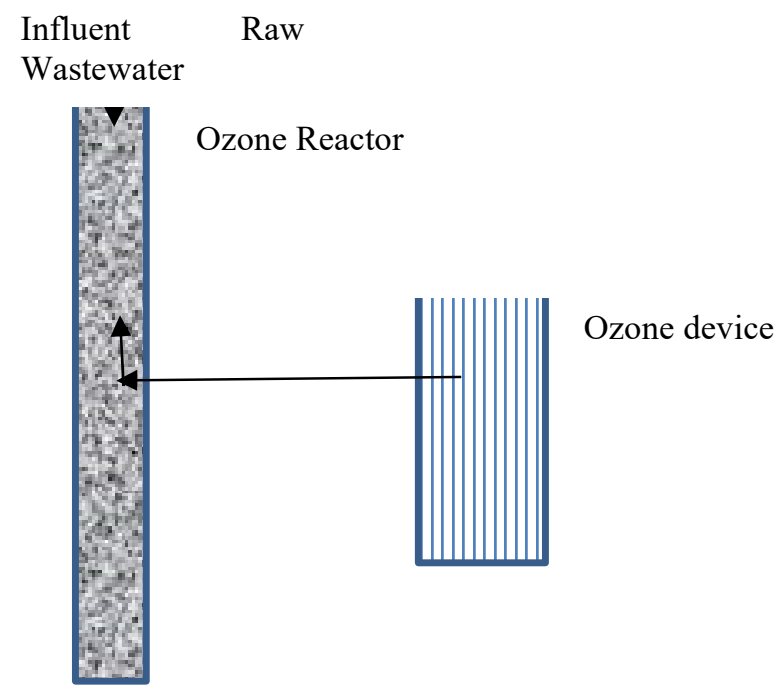

Figure 1. Ozonation System (Sequential Batch)

0.38\% 7-Hydroxy-7-phenly-3,9-diisopropyl-2,10-dioxadispirı, 1.71\% 2-Propanone(CAS) Acetone, 20.87\% 2-propanol,2-methyl-(CAS) tert-Butyl alcohol, 14.92\% 2-Butanone(CAS) Methyl ethyl ketone, 62.11\% Hydroperoxide,1,1-dimethyl(CAS). Cadox TBH parameters were measured in the wastewater samples taken from the balancing tank. In addition, the water contained 17,000 mg / 1 COD and very low total nitrogen $(<6.2)$.

Intermediate product profile were determined by Shimadzu GCMS-QP2020. The ozonation was carried out for seven hours. Palintest interface photometer 7500(COD kit no:PL454, wtw cr2200 termoreactor) and WTW340 $\mathrm{pH}$ meter were used in the measurements of COD and $\mathrm{pH}$. All measurements were performed according to standard methods (Standart Methods, 1995).

\section{Results}

In the present study, wastewater from the equalization tank was subjected to ozonation for seven hours. The $\mathrm{pH}$ value of the wastewater decreases depending on the ozonation time. As a result of ozonation, the influent COD value decreased from $17,000 \mathrm{mg} / 1$ to $2,860 \mathrm{mg} / 1$. As a result of influent wastewater GS-MS analysis, it was found that it contained $0.38 \%$ Hydroxy-7-phenyl-3,9-diisopropyl-2,10-dioxadispirol, $1.71 \% \quad 2$ propanone(Acetone), 20.87\% 2-propanol,2-methyl(tert-butyl alcohol), 14.92\% 2-Butanone(Methyl ethyl ketone), and $62.11 \%$ Hydroperoxide, 1,1-dimethylethyli.

The intermediate product profile after one hour of ozonation is shown in Figure 2. The wastewater contained 
Hydroxy-7-phenyl-3,9-diisopropyl-2,10-dioxadispirol(A), 2-propanone(Acetone)(B), 2-propanol,2-methyl(tertbutyl alcohol)(C), 2-Butanone(Methyl ethyl ketone)(D), Acetic acid (Ethylic Acid)(E), 2-Butanol, 2-methyl-( 2Methyl-2- butanol)(F), Hydroperoxide, 1,1-dimethylethyl(G), and Nitrogen oxide(H) as a result of one hour ozonation. After one hour of ozonation, three new intermediates (Acetic acid (Ethylic Acid) (E), 2-Butanol, 2methyl- (2-Methyl-2-butanol) (F), and Nitrogen oxide (H)) were formed. Hydroxy-7-phenyl-3,9-diisopropyl-2,10dioxadispirol (A), which was $0.38 \%$ in the influent wastewater, was removed from the medium after one hour of ozonation. Hydroperoxide, 1,1-dimethylethyl $(\mathrm{G})$ decreased from $62.11 \%$ to $46.61 \%$. In addition, 2-butanone (methyl ethyl ketone) (D) decreased from $14.92 \%$ to $4.82 \%$. This shows that the $\mathrm{C}-\mathrm{C}$ and $\mathrm{C}=\mathrm{O}$ bonds in the ketone structure were rapidly disintegrated by ozone. Nitrogen oxide content in the wastewater increased to $4.04 \%$. Compared to Figures 2 and 8, organic matter removal efficiency was found to be $53 \%$.

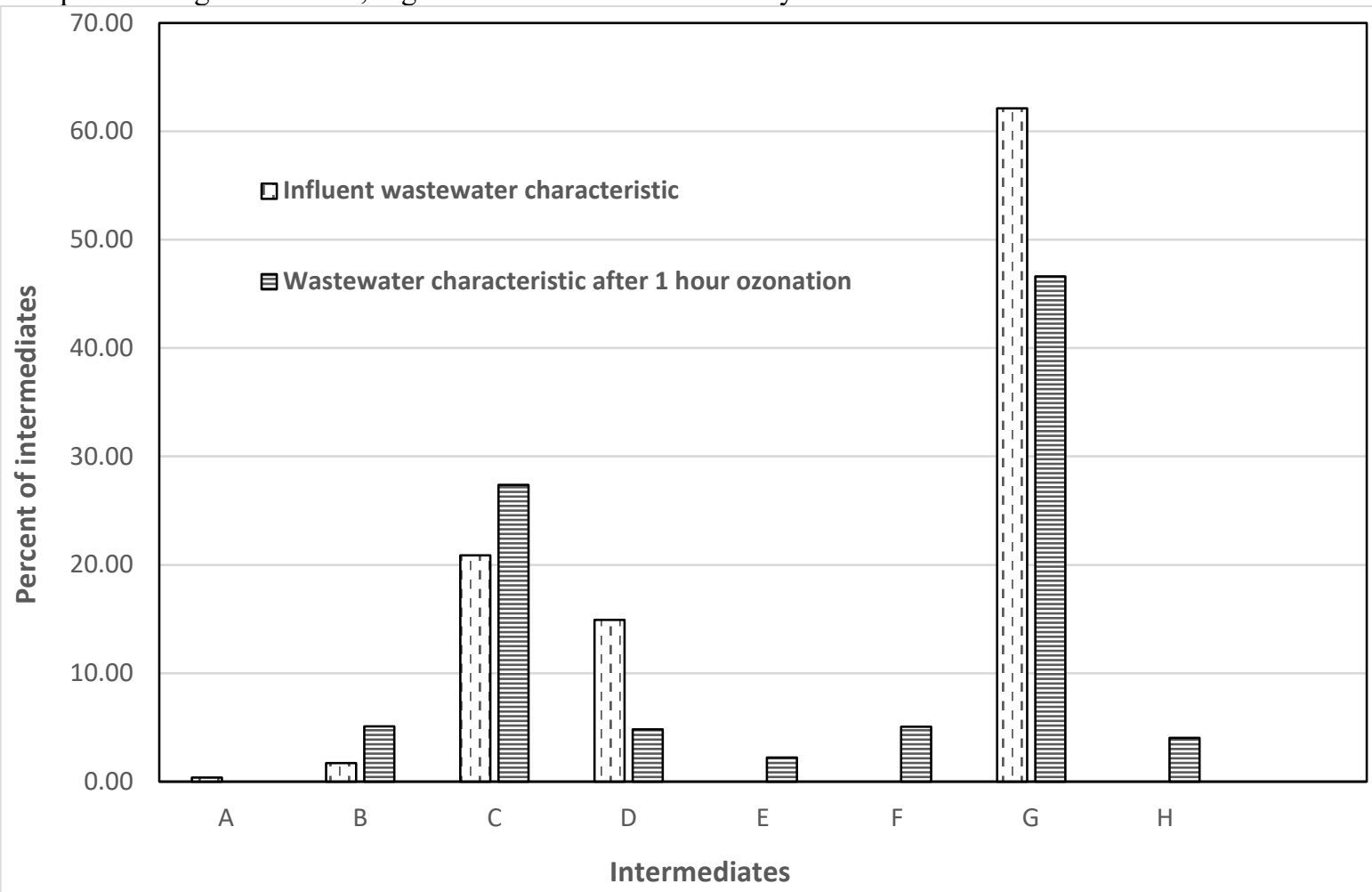

Figure 2. Intermediate product change with one hour ozonation

The intermediate product profile after three hours of ozonation is shown in Fig. 3. After three hours of ozonation, the wastewater characteristics changed to Hydroxy-7-phenyl-3,9-diisopropyl-2,10-dioxadispirol (A), 2-propanone(Acetone) (B), 2-propanol,2-methyl(tert-butyl alcohol) (C), 2-Butanone(Methyl ethyl ketone) (D), Acetic acid (Ethylic Acid) (E), 2-Butanol, 2-methyl-(2-Methyl-2-butanol) (F), Hydroperoxide, 1,1-dimethylethyl (G), Nitrogen oxide (H), N,2-Dimethydodecylamine (K), Propanoic acid, 2-hydroxy-2-methyl(hydroxybutyric) (L), 2-Butanone, 3-hydroxy-3 methyl (M), (N,2-Dimethydodecylamine (K) (4.29\%), Propanoic acid, 2-hydroxy2-methyl (hydroxybutyric) (L) (3.06\%), and 2-Butanone, 3-hydroxy-3 methyl (M) (2.81\%). After three hours, $49 \%$ Hydroperoxide $(\mathrm{G})$ removal was achieved. The concentration of 2-butanol, 2-methyl- (2-Methyl-2-butanol (F) decreased from $5.06 \%$ to $2.51 \%$. Methyl ethyl ketone (D) concentration decreased by $53 \%$. Acetone (B) formation increased in the medium with ozone (from $5.10 \%$ to $16.17 \%$ ). Also, the concentration of tert-butyl alcohol (C) increased with the breakdown of complex organic substances. The organic substances were degraded by ozone and formed acetic acid (E). In addition, after three hours of ozonation, 73\% of the organic matter was oxidized to the end products. $27 \%$ of the intermediate product was formed. 


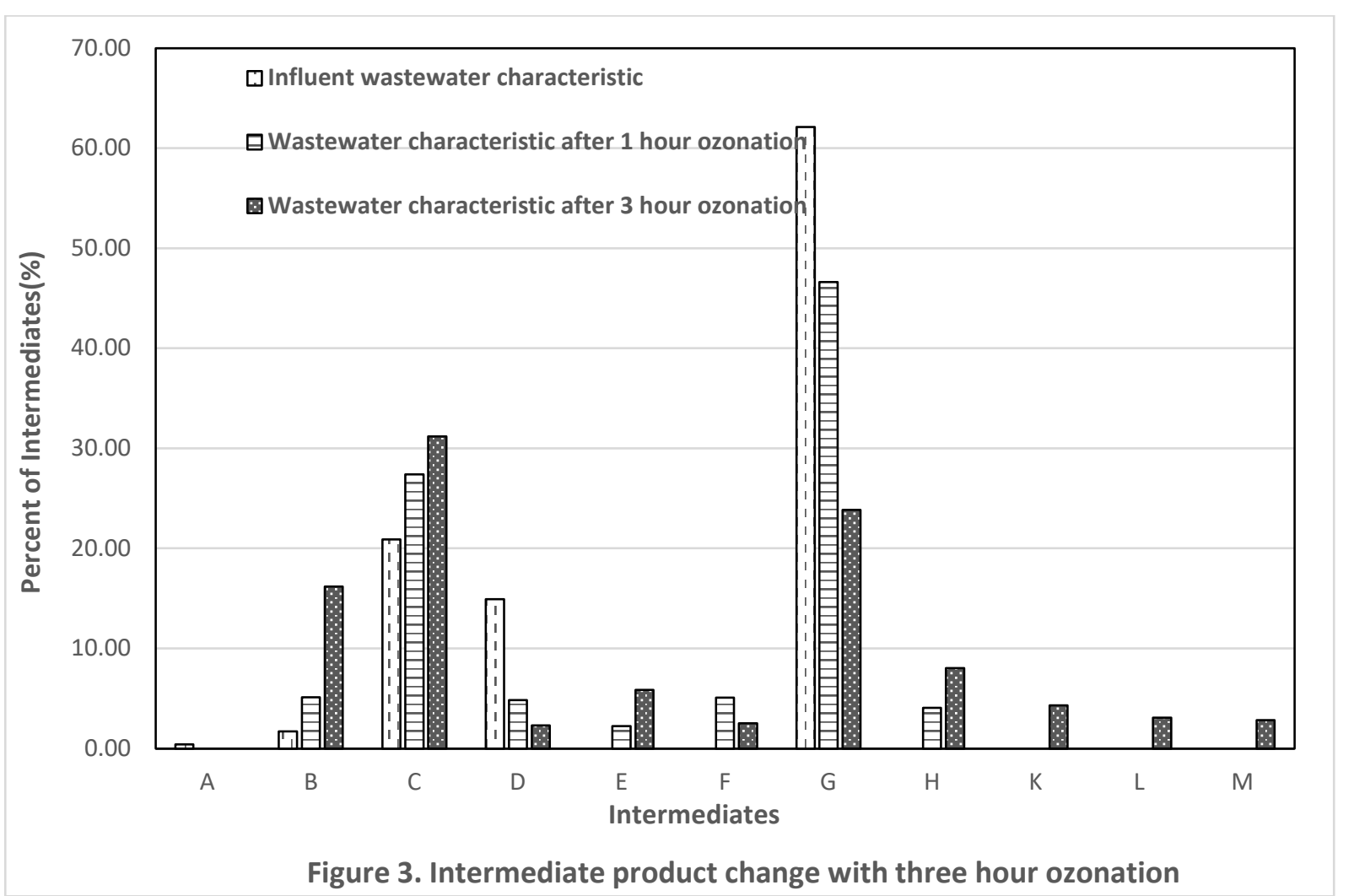

After four hours of ozonation, 2-propanone (Acetone) (B) (24.92\%), 2-propanol, 2-methyl (tert-butyl alcohol) (C) $(32.81 \%)$, Acetic acid (Ethylic Acid) (E) (8.54\%), 2-Butanol, 2-methyl- (2-Methyl-2-butanol) (F) (1.56\%), Hydroperoxide, 1,1-dimethylethyl (G) (11.36\%), Nitrogen oxide (H) (9.23\%), Propanoic acid, 2-hydroxy-2methyl (hydroxysibutyric) (L) (3.49\%), 2-Butanone, 3-hydroxy-3 methyl (M) (5.13\%), and 2,3 Butanedione (diacetyl) $(\mathrm{N})(1.06 \%)$ were measured in the medium. After four hours of ozonation, 2,3 Butanedione (diacetyl) $(\mathrm{N})$ intermediate was formed. Intermediate 2-Butanone (Methyl ethyl ketone) (D) and N, 2-Dimethydodecylamine $(\mathrm{K})$ were removed from the medium at the end of the fourth hour. There was an increase in the percentages of 2propanone (Acetone) (B), 2-propanol, 2-methyl (tert-butyl alcohol) (C), Acetic acid (Ethylic Acid) (E), Nitrogen oxide $(\mathrm{H})$ Propanoic acid, 2-hydroxy-2-methyl (hydroxybutyric) (L), and 2-butanone, 3-hydroxy-3 methyl (M) at the end of ozonation. In contrast, intermediate concentrations of 2-butanol, 2-methyl- (2-Methyl-2-butanol) (F), and Hydroperoxide, 1,1-dimethylethyl (G) decreased. As seen in Figure 4, mainly 2-propanone (Acetone) (B), 2propanol, 2-methyl (tert-butyl alcohol) (C), and Acetic acid (Ethylic Acid) (E) formation was observed. Methylene chloride, oxalic acid, acetone, etc. are resistant to hydroxyl radicals(Dulov et al.,). As compared to Fig. 4 and Fig. 8 , after three and four hours of ozonation, it was found that the existing wastes were converted into other intermediate products and did not break up to the final products (COD removal efficiency was $1.3 \%$ ). 


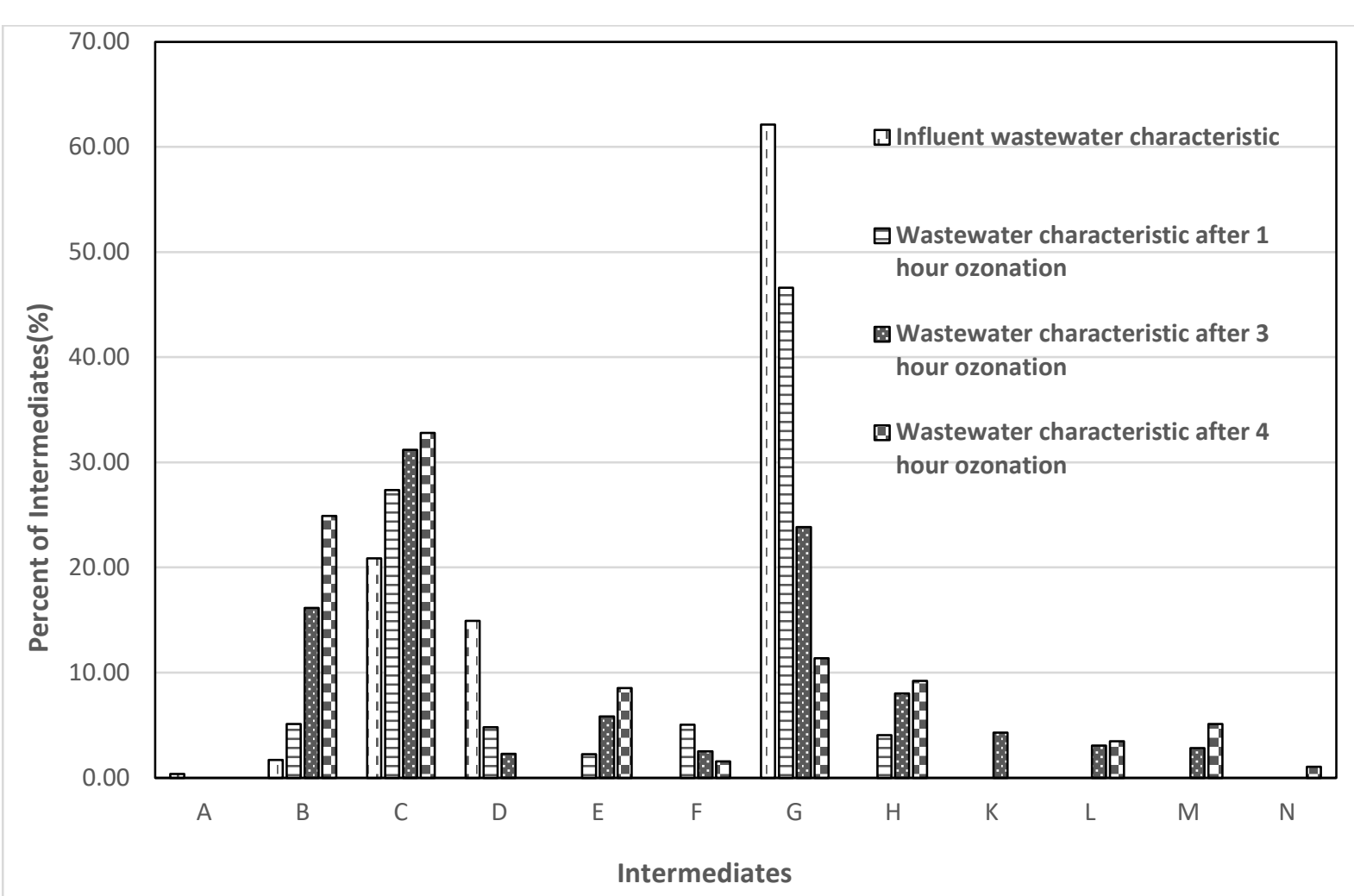

Figure 4. Intermediate product change with four hour ozonation

After five hours ozonation, 2-propanone (Acetone) (B), 2-propanol, 2-methyl (tert-butyl alcohol) (C), 2Butanone (Methyl ethyl ketone) (D), Acetic acid (Ethylic Acid) (E), Hydroperoxide, 1,1-dimethylethyl (G), Nitrogen oxide (H), Propanoic acid, 2-hydroxy-2-methyl (hydroxybutyric) (L), 2-Butanone, 3-hydroxy-3 methyl (M), 2,3 Butanedione (diacetyl) $(\mathrm{N})$ and Ammonium bicarbonate (O) intermediates were detected. 2-Butanol, 2methyl- (2-Methyl-2-butanol) $(\mathrm{F})$ was removed at the end of the ozonation. In addition, ammonium bicarbonate (O) $(4.95 \%)$ intermediate product formation was observed at the end of this period. The concentration of 2propanone (Acetone) (B), which was $1.71 \%$ in the influent wastewater, increased to $32.84 \%$ after five hours. The concentrations of 2-propanol, 2-methyl (tert-butyl alcohol) (C) and acetic acid (Ethylic Acid) (E) increased to 28.1\% and $11.48 \%$, respectively. Hydroperoxide, 1,1-dimethylethyl $(\mathrm{G})$ concentration decreased from $11.36 \%$ to $2.3 \%$. Butanone (Methyl ethyl ketone) (D), which disappeared in the medium at the fourth hour, re-formed by $1.22 \%$. Nitrogen oxide (H), Propanoic acid, 2-hydroxy-2-methyl(hydroxybutyric) (L), 2-Butanone, 3-hydroxy-3 methyl (M) and 2,3 Butanedione(diacetyl) (N) products increased or decreased in limited amounts. Fig. 5 and Fig. 8 show that predominantly 2-propanone (Acetone) (B) and 2-propanol, 2-methyl (tert-butyl alcohol) (C) were formed. The COD removal efficiency was $36 \%$ between four and five hours. As a result of the ozonation, the organic materials in the wastewater have been partially disintegrated into the final products. 


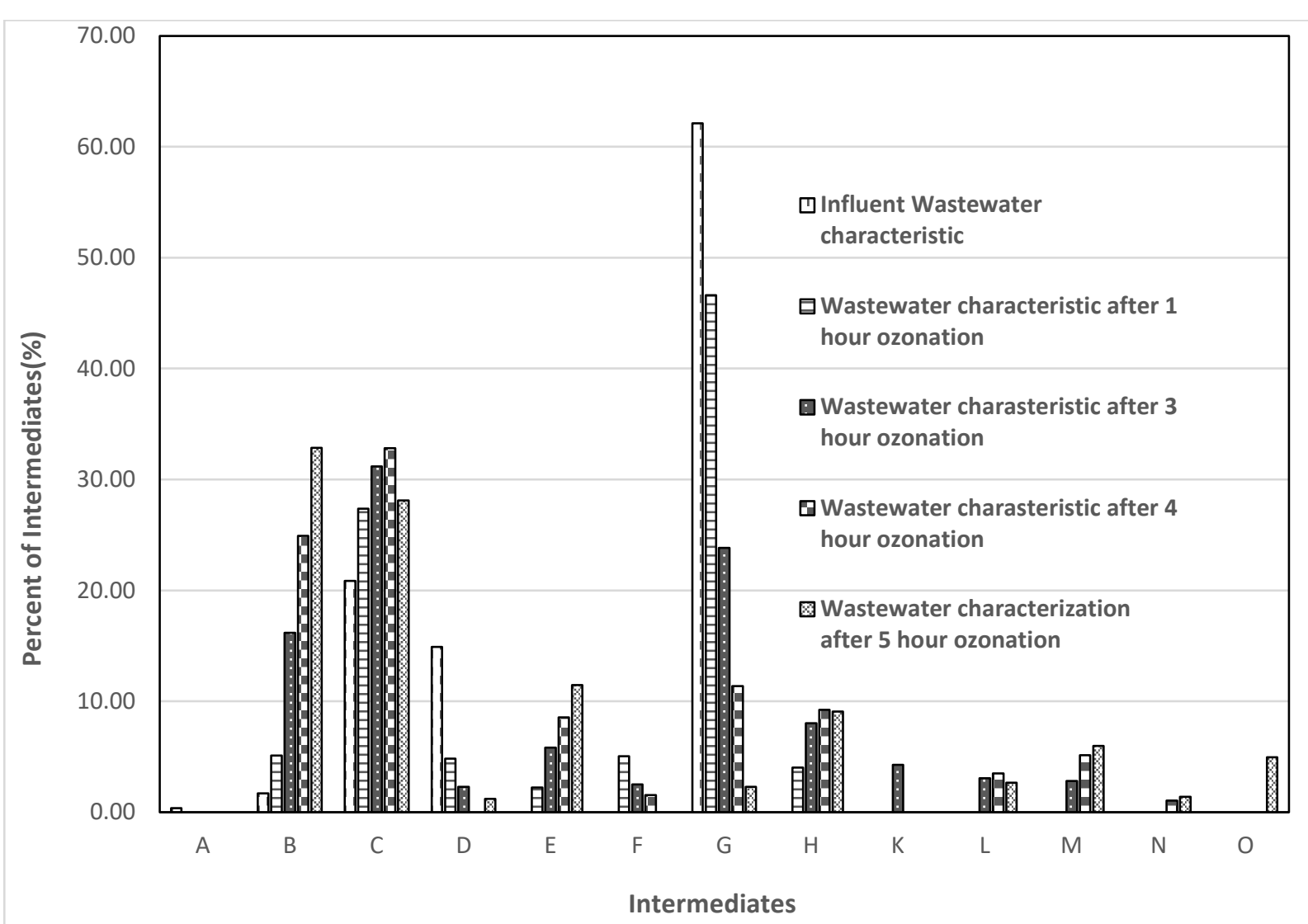

Figure 5. Intermediate product change with five hour ozonation

At the end of the six hour ozonation, 2-propanone (Acetone) (B), 2-propanol, 2-methyl (tert-butyl alcohol) (C), 2-Butanone (Methyl ethyl ketone) (D), Acetic acid (Ethylic Acid) (E), Nitrogen oxide (H), 2-Butanone, 3hydroxy-3 methyl (M), 2,3 Butanedione (diacetyl) (N), Carbamic acid, monoammonium salt (Ammonium Carbamat) (P), and Acetic acid, ethoxy-, 1-methylethyl ester (R) were found in ozonated wastewater. The concentration of 2-propanone (Acetone) (B) increased to $38 \%$ at the end of the sixth hour. The concentrations of Hydroperoxide, 1,1-dimethylethyl (G), Propanoic acid, 2-hydroxy-2-methyl (hydroxysibutyric) (L) and Ammonium bicarbonate $(\mathrm{O})$ decreased from $2.3 \%, 2.67 \%$ and $4.95 \%$ to zero, respectively. In contrast, Carbamic acid, monoammonium salt (Ammonium Carbamat) (P), and Acetic acid, ethoxy-, 1-methylethyl ester (R) concentrations increased from zero to $4.08 \%$ and $1.25 \%$, respectively. The concentration of 2-propanol, 2-methyl (tert-butyl alcohol) (C) decreased by $2.34 \%$. The concentration of 2-butanone, 3-hydroxy-3 methyl (M) increased from $5.97 \%$ to $7.87 \%$. Small decreases or increases in 2-butanone (Methyl ethyl ketone) (D), Acetic acid (Ethylic Acid) (E), Nitrogen oxide $(\mathrm{H})$ and 2,3 Butanedione (diacetyl) $(\mathrm{N})$ concentrations were determined. Compared to Fig. 6 and Fig. 8, the COD removal efficiency was 55\% between the fifth and sixth hours. 


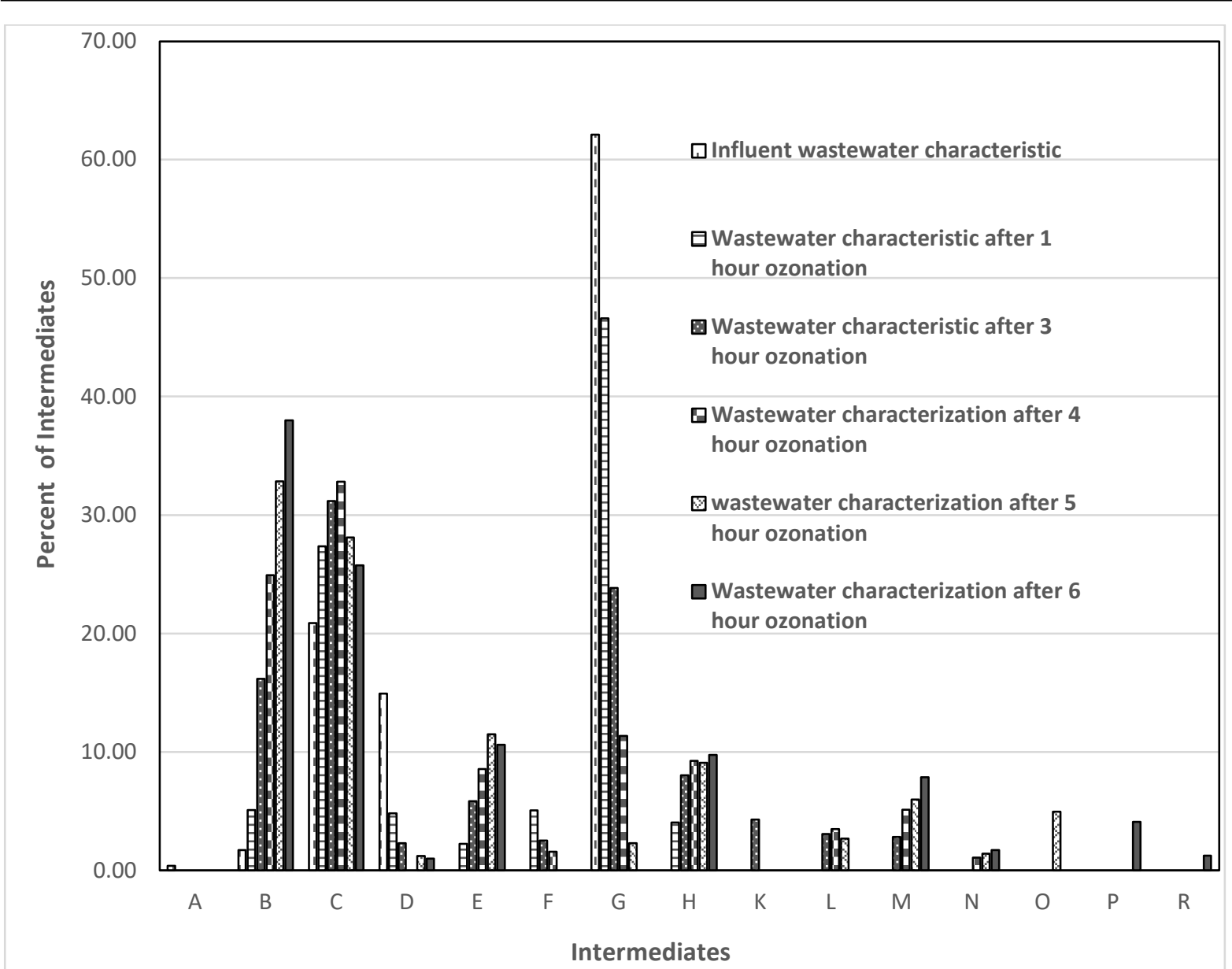

Figure 6. Intermediate product change with one hour ozonation

After seven hours of ozonation, the following product profile was found: 2-propanone (Acetone) (B) $41.52 \%$, 2-propanol, 2-methyl (tert-butyl alcohol) (C) 23.19\%, 2-butanone (D) $0.76 \%$, Acetic acid (Ethylic Acid) (E) 7.28\%, Nitrogen oxide (H) 10.54\%, 2-Butanone, 3-hydroxy-3 methyl (M) 8.41\%, and 2,3 Butanedione (diacetyl) (N) 2.04\%. Carbamic acid, monoammonium salt (Ammonium Carbamat) (P), Acetic acid, ethoxy-, and 1-methylethyl ester $(\mathrm{R})$ formed after six hours of ozonation were completely removed from the medium after seven hours. At the end of the seventh hour, no new intermediates were formed in the ozonation process. At the end of the sixth and seventh hours, the concentrations of some products increased (2-propanone (Acetone) (B) from 38\% to 41\%, Nitrogen oxide (H) from $9.74 \%$ to $10.54 \%$, 2-Butanone, 3-hydroxy-3 methyl (M) from 5.97\% to $7.87 \%$, and 2,3 Butanedione (diacetyl) (N) from 1.71\% to 2.04\%). In contrast, 2-propanol, 2-methyl (tert-butyl alcohol) (C) decreased from $25.76 \%$ to $23.19 \%$, 2-Butanone (Methyl ethyl ketone) (D) from $1 \%$ to $0.76 \%$, Acetic acid (Ethylic Acid (E) from $10.59 \%$ to $7.28 \%$, Carbamic acid, monoammonium salt (Ammonium Carbamat) (P) from $4.08 \%$ to $0 \%$ and Acetic acid, ethoxy-, 1-methylethyl ester (R) from $1.25 \%$ to $0 \%$. Acetone formation was observed during this period. Figure 7 and Figure 8 show that the final product formation was limited (23\%) as a result of ozonation. Due to the formation of intermediates such as acetone, 2-butanone, 3-hydroxy-3 methyl (M), and 2,3 butanedione (diacetyl) $(\mathrm{N})$, the formation of final $\left(\mathrm{CO}_{2}, \mathrm{NO}_{2}\right)$ products was limited. $\mathrm{COD}$ removal efficiency was $23 \%$ between the sixth and seventh hours. According to the influent COD concentration, at the end of the seventh hour the substances and intermediate products were converted into the final products at a rate of $94 \%$. 


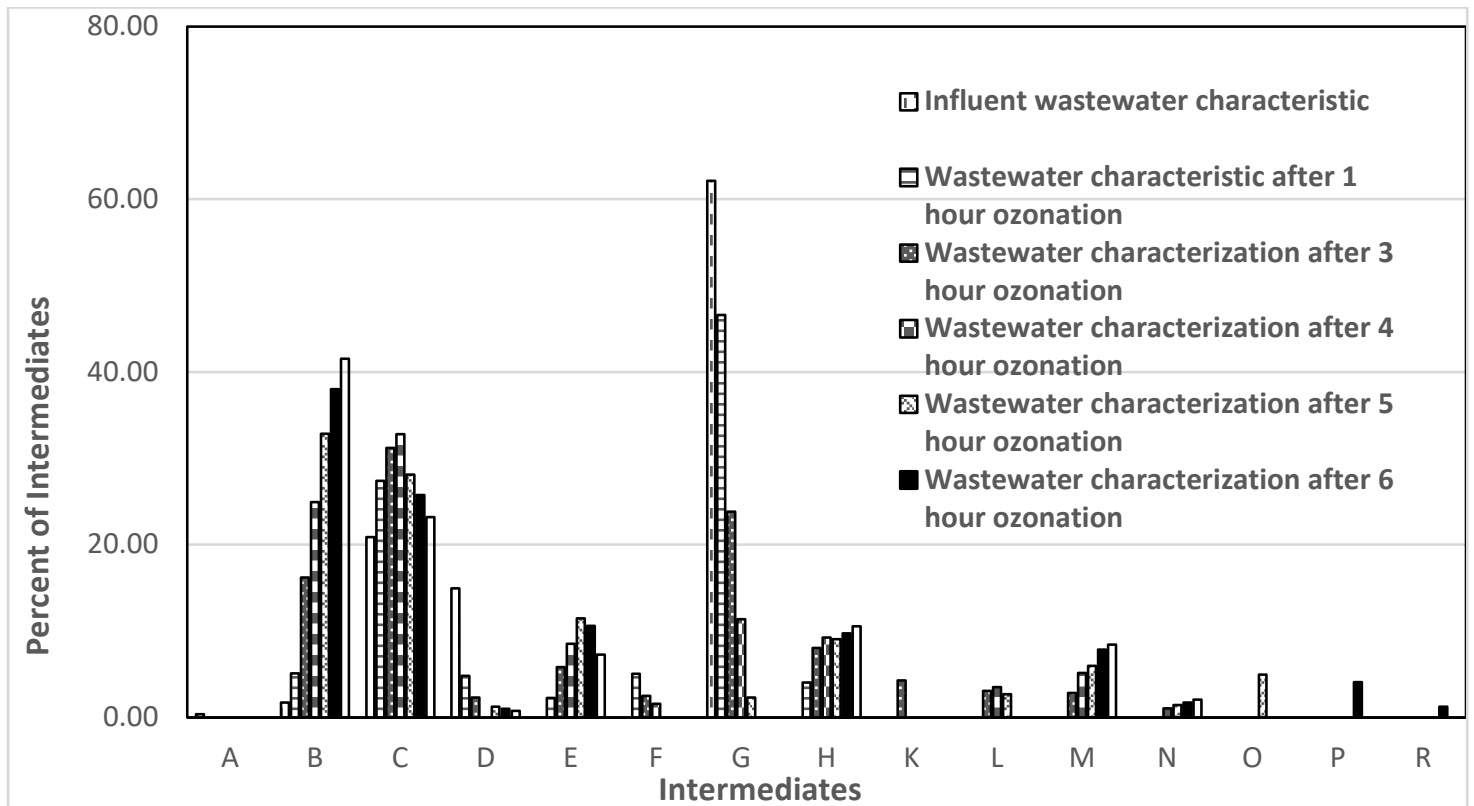

Figure 7. Intermediate product change with seven hour ozonation

As shown in Fig. 8, the effluent COD values decreased according to the ozonation time (ozone concentration). At the end of the first hour, the COD value decreased to $8,010 \mathrm{mg} / \mathrm{l}$. At the end of three, four, five, six and seven hours, the COD concentration decreased to 4,520,4,460, 2,860 and 1,000 mg / 1, respectively. At the end of the seventh hour, COD removal efficiency was $94 \%$. As can be seen from COD removal, large parts of the influent and intermediate products are broken down into the final products by ozonation. The COD removal efficiency remained constant between three and four hours. Acetone, tert-butyl alcohol and acetic acid, which increased in concentration at the end of the third hour, showed that they did not immediately break down. In addition, new intermediate N, 2-Dimethydodecylamine (K), Propanoic acid, 2-hydroxy-2-methyl (hydroxybutyric) (L), and 2butanone, 3-hydroxy-3 methyl (M), were observed in this range. There was an increase in the rate of nitrogen oxide between four and seven hours and a decrease in some intermediate product formation.

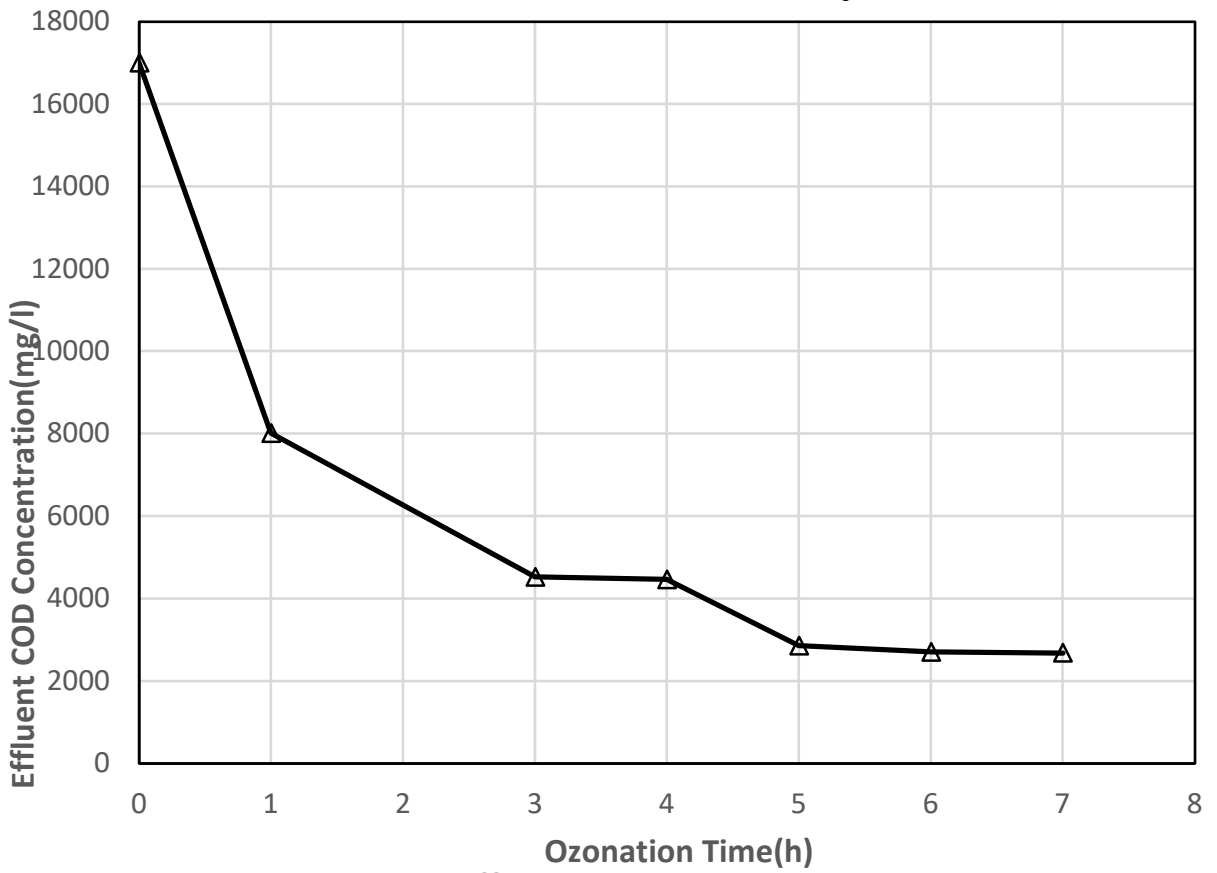

Figure. 8. Effluent COD Change with Ozonation Time

\section{Conclusion}

In this study, the oxidation of wastewater containing organic peroxide with ozone and the formation of intermediate product were investigated. Also, COD removal efficiencies were determined depending on the ozonation time. It 
was found that the influent wastewater contained: Hydroxy-7-phenyl-3,9-diisopropyl-2,10-dioxadispirol (A) 0.38\%, 2-propanone(Acetone) (B) 1.71\%, 2-propanol,2-methyl(tert-butyl alcohol) (C) 20.87\%, 2-Butanone (Methyl ethyl ketone) (D) 14.92\%, and Hydroperoxide, 1,1-dimethylethyl (G) 62.11\%. Depending on the length of ozonation, some new intermediates were formed while others were completely destroyed. After seven hours of ozonation, Hydroxy-7-phenyl-3,9-diisopropyl-2,10-dioxadispirol (A), 2-Butanol, 2-methyl- (2-Methyl-2-butanol) (F), Hydroperoxide, 1,1-dimethylethyl (G), N, 2-Dimethydodecylamine (K), Propanoic acid, 2-hydroxy-2-methyl (hydroxybutyric) (L), Ammonium bicarbonate (O), Carbamic acid, monoammonium salt (Ammonium Carbamat) $(\mathrm{P})$ and Acetic acid, ethoxy-, 1-methylethyl ester (R) were removed from the medium. In addition, the ratio of 2propanone (Acetone) (B) increased from 1.71\% to $41.52 \%$. At the end of the fifth and sixth hours, three new intermediates (Ammonium bicarbonate, Carbamic acid, monoammonium salt, Acetic acid, ethoxy-, 1-methylethyl ester) were formed. After seven hours of ozonation, these products were completely removed from the medium.

\section{References}

Aparicio,M.A., Eiroa,M., Kennes,C., Veiga, M.C.(2007). Combined post-ozonation and biological treatment of recalcitrant wastewater from a resin-producing factory. Journal of Hazardous Materials. 143, 285-290.

Asghar, A., Raman, A.A.A., Daud, W.M.A.W.(2015). Advanced oxidation processes for in-situ production of hydrogen peroxide/hydroxyl radical for textile wastewater treatment: a review. J. Clean. Prod. 87, 826-838.

Bourgin,M., Beck,B., Boehler, M., Borowska,E., Fleiner, J., Salhi,E., Teichler,R., von Gunten,U., Siegrist,H., McArdell,C.(2018). Evaluation of a full-scale wastewater treatment plant upgraded with ozonation and biological post-treatments: Abatement of micropollutants, formation of transformation products and oxidation by-products. Water Research, 129 (2018), 486-498.

De Souza, S.M.D.A.G.U., Bonilla,K.A.S., de Souza,A.A.U.(2010). Removal of COD and color from hydrolyzed textile azo dye by combined ozonation and biological treatment, J. Hazard. Mater. 179(1-3), 35-42.

Dulov, A.,Dulova, N.,Trapido, N.(2011). Combined Physicochemical Treatment of Textile and Mixed Industrial Wastewater. Ozone: Science \& Engineering.33, 285-293.

Gomes,J., Costa,R., Quinta-Ferreira,R.M., Martins,R.C.(2017). Application of ozonation for pharmaceuticals and personal care products removal from water, Sci. Total Environ. 586, 265-283.

Gunukula,R.V.B., Tittlebaum, M, E.(2001). Industrial wastewater treatment by an advanced oxidation process. Journal of Environmental Science and Health. 36(3), 307-320.

Hollender, J., Zimmermann, S.G., Koepke, S., Krauss, M., McArdell, C.S., Ort, C., Singer, H.,Gunten, H.V., Siegrist, H. ( 2009). Elimination of organic micropollutants in a municipal wastewater treatment plant upgraded with a full-scale post-ozonation followed by sand filtration. Environmental Science \& Technology. 43(20), 7862-7869

Lee, C.O., Howe, K.J., Thomson, B.M. (2012). Ozone and biofiltration as an alternative to reverse osmosis for removing PPCPs and micropollutants from treated wastewater. Water Res. 46(4),1005- 1014.

Mehrjouei,M., Müller,S., Möller,D.(2015). A review on photocatalytic ozonation used for the treatment of water and wastewater. Chemical Engineering Journal. 263, 209-219.

Papageorgiou,A., K. Stylianou,S., Kaffes,P., I. Zouboulis,A., Voutsa, D.(2017). Effects of ozonation pretreatment on natural organic matter and wastewater derived organic matter e Possible implications on the formation of ozonation by-products. Chemosphere 170, 33-40.

Standard Methods for the Examination of Water and Wastewater.(1995). 18 ${ }^{\text {th }}$ Edition, Washington Dc, USA,1995.

Turhan, K., Durukan, I., Ozturkcan, S.A., Turgut, Z.(2012). Decolorization of textile basic dye in aqueous solution by ozone. Dyes Pigment. 92, 897-901. 